\title{
Problemas Emocionais e de Comportamento e Clima Familiar em Adolescentes e seus Pais
}

\author{
Maycoln Leôni Martins Teodoro \\ Universidade Federal de Minas Gerais \\ Minas Gerais, BH, Brasil \\ Adriana Raquel Binsfeld Hess \\ Lisiane Alvim Saraiva \\ Universidade Federal do Rio Grande do Sul \\ Porto Alegre, RS, Brasil \\ Bruna Moraes Cardoso \\ Universidade Regional Integrada do Alto Uruguai e das Missões \\ Erechim, RS, Brasil
}

\begin{abstract}
RESUMO
A adolescência é uma fase de mudanças e um período vulnerável para o desenvolvimento de problemas emocionais e de comportamento (PEC). Estes podem surgir em decorrência de crises familiares, consideradas fatores de risco. Este estudo investigou os PEC e sua relação com aspectos do clima familiar na visão de 187 adolescentes e suas mães e pais. Os jovens eram estudantes de escolas públicas do Rio Grande do Sul. Os instrumentos utilizados foram o Youth SelfReport (YSR), o Adult Self-Report (ASR) e o Inventário do Clima Familiar (ICF). Os resultados das percepções dos participantes quanto aos sintomas internalizantes e externalizantes indicaram relação da descrição das mães e pais e das mães e filhos, mas não entre descrições de filhos e pais. Os problemas internalizantes maternos correlacionaram-se positivamente com a percepção de Hierarquia dos adolescentes e negativamente com a Afetividade e os externalizantes correlacionaram-se positivamente com Conflito e Hierarquia, e negativamente com Coesão e Afetividade.
\end{abstract}

Palavras-chave: Problemas do comportamento; adolescência; estrutura familiar.

\section{ABSTRACT}

\section{Emotional and Behavioral Problems and Family Climate in Adolescents and their Parents}

Adolescence is a period of many changes and a vulnerable phase for the development of emotional and behavioral problems (EBP). These may happen due to family crises, considered as risk factors. This study investigated EBP and their relation with family climate in the view of 187 adolescents and their mothers and fathers. The youngsters were students from public schools in Rio Grande do Sul. The used instruments were Youth Self-Report [YSR], Adult SelfReport [ASR] and Family Climate Inventory. The results of the participants' perceptions, considering internalizing and externalizing symptoms, indicated a relation between mothers and fathers' and mothers and children's descriptions, but not between fathers and children's. The maternal internalizing problems were positively correlated with the perceived Hierarchy of adolescents and negatively with Affection; the externalizing problems were positively correlated with Conflict and Hierarchy, and negatively with Cohesion and Affection.

Keywords: Behavioral problems; adolescence; family structure (or family).

\section{RESUMEN}

Problemas Emocionales y de Comportamiento y el Clima Familiar para los Adolescentes y sus Padres

La adolescencia es una fase de cambios y un periodo vulnerable para el desarrollo de problemas emocionales y de comportamiento (PEC). Estos pueden aparecer en consecuencia de crisis familiares, consideradas como factores de riesgo. Esta investigación abordó los PEC y su relación con los aspectos de un clima familiar en el enfoque de 187 adolescentes y sus padres. Los jóvenes fueron estudiantes de escuelas publicas de Rio Grande do Sul. Los instrumentos utilizados fueron YSR, ASR y ICS. Los resultados de las percepciones de los participantes en relación a los síntomas internalizantes y externalizantes indican relación de la descripción entre madres y padres, y de las madres e hijos, pero no aborda descripciones de hijos e padres. Los problemas internalizantes maternos se correlacionan positivamente con la percepción de la Jerarquía de los adolescentes y negativamente con la Afectividad, y los externalizantes se relacionan positivamente con Conflicto y Jerarquía, y negativamente con Cohesión y Afectividad.

Palabras clave: Problemas de comportamiento; adolescencia; estructura familiar. 
A adolescência é uma fase na qual há uma exacerbação das exigências emocionais e comportamentais sofridas pelos indivíduos (Buehler, 2006). É um período em que ocorrem concomitantemente mudanças biológicas, psicológicas, culturais e psicossociais, caracterizando-se, assim, como a principal fase de consolidação da identidade (Parsons, 2003). Trata-se de uma etapa de transição da infância para a idade adulta, na qual o indivíduo deve evoluir de um estado de extrema dependência para outro de maior autonomia pessoal. É, portanto, um momento do ciclo vital individual que apresenta simultaneamente tarefas particulares e tarefas que envolvem todos os membros da família (Pratta \& Santos, 2007).

Para os pais, a adolescência dos filhos se mostra, frequentemente, um período difícil e desafiador. Oliva e Parra (2004) revisam diversos autores e concluem que durante a adolescência dos filhos a maioria das famílias, mesmo aquelas que até então haviam tido relações predominantemente harmoniosas, atravessa uma época de desgastes, desajustes e maiores conflitos. A comunicação entre pais e filhos, assim, pode se deteriorar em algum momento e provocar mudanças nos padrões de interação. A família precisa se flexibilizar e se adaptar a um novo funcionamento, oferecendo um ambiente que promova o crescimento e desenvolvimento dos filhos adolescentes. O papel dos adultos é fundamental neste processo, pois são eles que devem prover a base inicial aos mais jovens, as regras e normas sociais, servindo como modelos a partir de ideias, atitudes e comportamentos transmitidos (Pratta \& Santos, 2007).

Face às transições típicas da faixa etária, a adolescência pode ser um período de vulnerabilidade para o surgimento de problemas emocionais e de comportamento (PEC) (Jacobs, Reinecke, Gollan, \& Kane, 2008). Os PEC na infância e adolescência não possuem consenso quanto à sua definição, havendo na literatura científica uma diversidade de conceituações que os designam. Neste estudo, adotou-se a definição proposta por Achenbach (1991), que caracteriza os PEC como sendo padrões sintomáticos, que podem ser divididos em dois tipos: externalizantes e internalizantes.

Os transtornos externalizantes são aqueles que se expressam em relação às outras pessoas e compreendem comportamentos como dificuldade em controlar impulsos, hiperatividade, agressividade e presença de raiva e delinquência (Achenbach \& Howell, 1993; APA, 2002). Os transtornos internalizantes são os que se expressam em relação ao próprio indivíduo e caracterizam-se pela tristeza, retraimento, queixas somáticas e medo (Achenbach \& Howell, 1993; APA, 2002).
Além das características individuais próprias da adolescência, estudos têm sugerido que aspectos familiares podem estar relacionados ao surgimento de PEC (Avanci, Assis, \& Oliveira, 2008; Cruvinel \& Boruchovitch, 2009; Hackett \& Hackett, 1999; Sheeber, Hops, Alpert, Davis, \& Andrews, 1997; Stark, Humphrey, Crook, \& Lewis, 1990; Teodoro, Cardoso, \& Freitas, 2010). Assim, muitos autores vêm investigando a relação entre PEC presentes nos adolescentes e a dinâmica do sistema familiar (Althoff, 2008; Costello, Rose, Swendsen, \& Dierker, 2008; Dorsey, Forehand, \& Brody, 2007; Harkness, Lumley, \& Truss, 2008; Kapi, Veltsista, Kavadias, Lekea, \& Bakoula 2007; Osório, 2002; Teodoro et al., 2010).

O campo das relações familiares é uma área de investigação de extrema importância para a compreensão do desenvolvimento psicológico (Benetti, 2006). O efeito do clima familiar no bem-estar psicológico dos adolescentes é tema recorrente em pesquisas atuais, sendo o conflito conjugal um dos reconhecidos fatores de risco para PEC nos adolescentes (Morawska \& Thompson, 2009), bem como as situações de crise familiar (Silvares \& Souza, 2008).

A manutenção da saúde familiar, no entanto, não depende apenas da capacidade de superar crises, mas, sobretudo, da boa qualidade das relações entre seus membros e das trocas familiares com o meio social (Pratta \& Santos, 2007). Assim, a falta de harmonia e baixa qualidade do relacionamento familiar e conjugal são aspectos importantes que influenciam diretamente o desenvolvimento dos filhos e podem culminar no aparecimento de dificuldades e na maior prevalência dos transtornos psicológicos e suas comorbidades nesta fase de vida dos indivíduos (Pratta \& Santos, 2007; Sawyer, Miller-Lewis, \& Clark, 2006).

Diversos estudos apontam para o impacto negativo dos conflitos conjugais no desenvolvimento psicológico dos filhos, especialmente nas situações familiares envolvendo violência física e verbal, manifestadas de forma coberta ou encoberta, entre o casal (Benetti, 2006; Teodoro, Cardoso, \& Pereira, 2011). Assim, os conflitos conjugais podem estar relacionados a distúrbios em diferentes aspectos do desenvolvimento de crianças e adolescentes, como nas áreas emocional, cognitiva e social (Benetti, 2006). Nesta perspectiva, a qualidade das relações familiares está associada à intensidade de PEC, sendo o relacionamento familiar saudável um dos muitos fatores associados com a saúde mental de seus membros. Existem, porém, outras características que também exercem influência no desenvolvimento de crianças e adolescentes, como genética, envolvimento com pares e condições socioeconômicas, que podem se configurar como fatores de risco relacionados 
ao surgimento de psicopatologias nesta faixa etária (Teodoro et al., 2011). Os resultados negativos gerados pela baixa qualidade das interações e do clima familiar podem estar presentes desde a infância, gerando transtornos emocionais e de comportamento somente na adolescência (Matud, 2007). Estudo longitudinal realizado pela autora analisou a associação entre abuso doméstico de mulheres, em suas múltiplas formas de maus tratos, sofrido por parte dos parceiros, e estressores internos e externos à família que tinham impacto na saúde das crianças, gerando repercussões também na adolescência. A amostra foi composta por 420 mulheres abusadas que tinham pelo menos um filho. Destas, $27,4 \%$ reportaram que um ou mais dos filhos apresentavam problemas psicológicos; $13,6 \%$ mencionaram problemas físicos de saúde apresentados pelas crianças; e 7,6\% relataram a presença de ambos. Os problemas externalizantes foram os mais frequentes, ocorrendo em $11,7 \%$ das crianças, enquanto os internalizantes estavam presentes em 5,6\% das mesmas. $(27,4 \%)$. Seus achados, portanto, sugerem uma forte associação entre maus tratos (abuso físico e sexual, negligência e exposição à violência doméstica) e estressores familiares (conflitos familiares, problemas emocionais e de comportamento dos pais e constrangimentos externos sobre a família) com o surgimento de PEC na adolescência.

Com relação a problemas ocorridos nas relações dos pais, Buehler, Benson e Gerard (2006) realizaram estudo com o objetivo de verificar o papel específico destes a partir da associação entre hostilidade interparental e a ocorrência de problemas precoces de comportamento em adolescentes. Através de uma amostra composta por 416 adolescentes de sexta série e seus pais (todos casados), contatados através de treze escolas situadas em um grande condado no sudeste dos Estados Unidos, os autores demonstraram que a intrusão materna mediou as associações entre hostilidade parental e problemas internalizantes e externalizantes de comportamento apresentados pelos filhos adolescentes. Esta relação se mostra mais significativa do que a paterna, principalmente no desenvolvimento de problemas externalizantes apresentados pelos jovens.

Tais dados são corroborados pelos achados de Adams e Laursen (2007), no que diz respeito à forte interação entre o adolescente e sua mãe. Em seu estudo, os autores examinaram se as associações entre os comportamentos adolescentes e os desentendimentos ocorridos com mães, pais e melhores amigos podiam variar em função da qualidade percebida do relacionamento. Os participantes foram 469 jovens entre 11 e 18 anos de idade. As qualidades positivas do relacionamento entre pais e adolescentes estavam ligadas às notas escolares e os problemas de ajustamento. Associações não lineares entre conflito e os comportamentos adolescentes foram moderadas por qualidades negativas de relações, de tal forma que os conflitos aumentaram de níveis baixos para moderados. Além desses fatores, o nível socioeconômico e sexo, também, foram preditivos para problemas de internalização e externalização.

Os PEC podem prejudicar, principalmente, o contado físico e emocional em relação à qualidade e a frequência (Fleitlich-Bilyk \& Goodman, 2001), o cuidado e o controle (Pedersen, 1994) e o respeito entre pais e filhos (Stocker, Richmond, \& Rhoades, 2007). Déficits na forma dos pais exercerem sua função adequadamente têm relação clara com PEC na infância e na adolescência. A qualidade da interação que os pais desenvolvem para com os filhos pode ser investigada, segundo Costello, Rose, Swendsen e Dierker (2008), por meio de sentimentos desenvolvidos pelo filho, em relação aos pais. Acrescenta-se, ainda, que tais sentimentos podem ser determinantes também no desenvolvimento das relações do mesmo com seus amigos, na escola e sua autoestima.

Apesar de grande parte das pesquisas comumente negligenciarem as influências do pai no desenvolvimento das crianças em contextos familiares e delegarem à mãe uma maior responsabilidade pela coesão familiar (Davies \& Epkins, 2009; EspositoSmytherset al., 2006), estudos recentes tem defendido a influência de ambos os pais no desenvolvimento dos filhos (Féres-Carneiro, Henriques, \& Jablonski, 2011; Marin, Piccinini, \& Tudge, 2011). Tal aspecto pode estar relacionado às mudanças que vem ocorrendo na sociedade, onde o pai tem sido cada vez mais solicitado a participar da educação dos filhos (Jablonski, 2010; Levandowski, Koller, \& Piccinini, 2002).

Tendo em vista o exposto, o objetivo deste estudo foi investigar os PEC e sua relação com aspectos do sistema familiar (clima familiar) na visão de adolescentes e seus pais. Considera-se de extrema importância o entendimento da relação entre PEC em adolescentes e aspectos do sistema familiar, a fim de que se possa contribuir para uma melhor identificação das potenciais metas de prevenção e de intervenção em nossa realidade.

\section{MÉTODO}

\section{Participantes}

Participaram do estudo 187 estudantes de duas escolas públicas de duas cidades do interior do Rio Grande do Sul, bem como suas mães e seus pais. A amostra foi composta por 112 participantes 
adolescentes do sexo feminino (59,9\%) e 75 do sexo masculino (40,1\%). A idade destes variou de 11 a 16 anos (Média $=12,8, D P=1,08)$. As 165 mães tinham idade média de 39,3 anos $(D P=6,8)$ e os 102 pais idade média de 42,4 anos $(D P=9,4)$.

\section{Instrumentos de Coleta de Dados}

Questionário Sociodemográfico. Todos os participantes do estudo (adolescentes e seus pais) preencheram um questionário com questões sociodemográficas, como profissão e escolaridade, e sobre a configuração familiar, como número de filhos e situação conjugal.

Inventário de Autoavaliação para Jovens de 11 a 18 anos (Youth Self-Report [YSR]). O YSR faz parte do Sistema de Avaliação Empiricamente Baseado (Achenbach System of Empirically Based Assessment - ASEBA), desenvolvido por Achenbach (Achenbach, 1991; Achenbach \& Rescorla, 2001). Trata-se de uma variação do Child Behavior Checklist - CBCL (Achenbach, 1991), na qual o respondente é o próprio adolescente. O YSR é uma escala Likert de três pontos ("não é verdadeira", "algumas vezes verdadeira" ou "muito verdadeira/frequentemente verdadeira") e está composto por oito escalas de problemas de comportamento: Ansiedade/Depressão, Isolamento/ Depressão, Queixas Somáticas, Problemas Sociais, Problemas de Pensamento, Problemas de Atenção, Comportamento de Quebra Regra e Comportamento Agressivo. Também compõe o instrumento um tópico denominado "Outros Problemas", que engloba itens que não se encaixam em nenhuma das outras escalas. Assim, o instrumento está composto da seguinte maneira: Problemas Internalizantes (incluem as três primeiras escalas), Problemas Externalizantes (incluem as duas últimas escalas) e Problemas Totais (inclui todas as escalas analisadas e/ou Outros Problemas). Neste estudo, foi utilizada a versão proposta por Rocha, Araújo e Silvares (2008), sendo utilizados os agrupamentos dos Problemas Externalizantes, composto pelos fatores Comportamento de Quebra Regra (alpha de Cronbach 0,62) e Comportamento Agressivo (alpha de Cronbach 0,85 ) e dos Problemas Internalizantes, formado pelos fatores Ansiedade/Depressão (alpha de Cronbach 0,80), Isolamento/Depressão (alpha de Cronbach 0,57), Queixas Somáticas (alpha de Cronbach 0,74).

Inventário de Autoavaliação para Adultos de 18 a 59 anos (Adult Self-Report [ASR]). O ASR é um inventário de autoavaliação que faz parte do ASEBA (Achenbach \& Rescorla, 2001; 2003) e avalia adultos de 18 a 59 anos de idade. No inventário estão incluídos 168 itens subdivididos em escalas sobre os Problemas de Comportamento Internalizante, Externalizante e Total. Os sintomas Internalizantes são formados pelos fatores Ansiedade/Depressão (alphas de Cronbach: 0,85/mães e 0,89/pais); Isolamento/Depressão (alphas de Cronbach: 0,65/mães e 0,73/pais) e Queixas Somáticas (alphas de Cronbach: 0,76/mães e 0,70/pais). Os externalizantes possuem os fatores Comportamento Agressivo (alphas de Cronbach: 0,86/mães e 0,87/pais); Quebra de Regras (alphas de Cronbach: $0,62 /$ mães e $0,73 /$ pais); e Comportamento Intrusivo (alphas de Cronbach: 0,64/mães e 0,64/pais).

Inventário do Clima Familiar - ICF. O ICF (Teodoro, Land, \& Allgayer, 2009) investiga o Clima Familiar por meio de 22 itens distribuídos em quatro construtos: Conflito, Hierarquia, Apoio e Conflito Familiar. O primeiro, Conflito, avalia a relação agressiva, crítica e conflituosa entre os membros da família (ex.: "Os conflitos são comuns", "As pessoas criticam umas as outras com freqüência”). O fator Hierarquia está relacionado a uma diferenciação rígida de poder dentro da família, na qual as pessoas mais velhas possuem influência impositiva nas decisões familiares. Representa o nível de poder e de controle dentro do sistema familiar (ex.: "É comum que algumas pessoas proibam outras de fazer determinadas coisas sem explicar o porquê"; "Uns mandam outros obedecem"). A dimensão Apoio contém itens que descrevem o suporte material e emocional dos membros. Avalia a existência de suporte emocional e material dado e recebido dentro da família (ex.: "Procuramos ajudar as pessoas da nossa família quando percebemos que estão com problemas"; "Quando alguém está doente, as outras cuidam dele”). Finalmente, a Coesão familiar foi definida como o vínculo emocional entre os membros da família. (ex.: "As pessoas sentemse felizes quando toda a família esta reunida"; "As pessoas gostam de passear e fazer coisas juntas"). Os alphas de Cronbach encontrados neste estudo variaram de 0,68 a 0,86 para os adolescentes, 0,70 a 0,89 para as mães e 0,60 a 0,87 para os pais.

\section{Procedimentos Éticos e de Pesquisa}

Inicialmente o projeto de pesquisa foi submetido ao Comitê de Ética da universidade de um dos autores. A partir da aprovação, realizou-se contato com a direção das escolas para apresentação da pesquisa. A partir do consentimento da direção, os alunos receberam a visita do pesquisador em sala de aula, ocasião na qual foram informados sobre os objetivos da pesquisa. Os alunos interessados em participar receberam uma carta destinada aos seus pais, contendo explicações sobre a pesquisa e o Termo de Consentimento Livre e Esclarecido (TCLE), que deveria ser assinado por um dos pais ou responsáveis autorizando o(a) filho(a) a participar. A partir do retorno dos TCLE, foi combinado 
com os professores o melhor horário para aplicação dos instrumentos nos adolescentes. Na data combinada, o pesquisador aplicou os instrumentos coletivamente na seguinte ordem, o Questionário Sociodemográfico, o YSR e o Inventário do Clima Familiar. Toda a aplicação durou, aproximadamente, 50 minutos. Após o término, os adolescentes receberam os instrumentos a serem preenchidos pelos pais em suas casas com as respectivas instruções. Quando concluído, os pais entregaram o material aos filhos que devolveram à escola, sendo que essa os repassou aos pesquisadores.

\section{Análise dos Dados}

Os dados foram analisados por meio de estatísticas paramétricas, sendo as associações entre variáveis analisadas através de Correlação de Pearson. Foi considerado como significativo todo resultado que obteve um $\mathrm{p}<0,05$.

\section{RESULTADOS}

\section{Correlações entre os Problemas Emocionais e de Comportamento dos Adolescentes e seus Pais}

As associações dos sintomas dos PEC dos adolescentes e de seus pais foram analisadas pelo coeficiente de correlação de Pearson. Os resultados estão descritos na Tabela 1.
De acordo com a Tabela 1, os coeficientes mais elevados foram aqueles entre os sintomas internalizantes e externalizantes, sendo $0,59(\mathrm{p}<0,001)$ para os adolescentes, $0,68(\mathrm{p}<0,001)$ para as mães e 0,79 $(\mathrm{p}<0,001)$ para os pais. Os sintomas internalizantes descritos pelas mães correlacionaram-se moderadamente correlacionados com a descrição dos pais $(\mathrm{r}=0,41$, $\mathrm{p}<0,001)$. Já a comparação dos sintomas externalizantes descritos pelas mães com o grupo paterno apresentou correlação fraca $(r=0,31, p<0,001)$. Os problemas emocionais e de comportamento dos filhos correlacionaram-se com os descritos pela mãe, com magnitudes de $0,37(p<0,01)$ para os internalizantes e $0,26(p<0,01)$ para os externalizantes. Não foi encontrada associação entre os sintomas dos filhos e dos pais.

\section{Correlações entre as Percepções do Clima Familiar dos Adolescentes e seus Pais}

As associações entre as diferentes perspectivas das relações familiares estão descritas na Tabela 2 . Com exceção das visões de apoio familiar entre pai e filho, todos os outros escores foram positivamente e significativamente correlacionados. Dentro da comparação mãe-filho, as associações significativas variaram de 0,35 (hierarquia) a 0,48 (apoio), enquanto para a díade pai-filho variou de 0,25 (conflito) a 0,32 (hierarquia e coesão). Para a díade mãe-pai, os escores foram de 0,28 (hierarquia) a 0,35 (coesão e conflito).

TABELA 1

Correlações entre os Problemas Emocionais e de Comportamento dos Adolescentes e de seus Pais

\begin{tabular}{lcccccc}
\hline & Inter.(A) & Exter.(A) & Inter.(M) & Exter.(M) & Inter. $(P)$ & Exter. $(P)$ \\
\hline Inter.(A) & - & - & - & - & - & - \\
Exter.(A) & $0,59^{* * *}$ & - & - & - & - & - \\
Inter.(M) & $0,37^{* * *}$ & $0,21^{* *}$ & - & - & - & - \\
Exter.(M) & $0,29^{* * *}$ & $0,26^{* *}$ & $0,68^{* * *}$ & - & - & - \\
Inter.(P) & 0,19 & 0,11 & $0,41^{* * *}$ & $0,39^{* * *}$ & - & - \\
Exter.(P) & 0,12 & 0,11 & $0,35^{* *}$ & $0,31^{* *}$ & $0,79^{* * *}$ & - \\
\hline
\end{tabular}

$* * \mathrm{p}<0,01, * * * \mathrm{p}<0,001$.

Abreviações: Inter.(A) (internalizantes dos adolescentes), Exter.(A) (externalizantes dos adolescentes), Inter.(M) (internalizantes das mães), Exter.(M) (externalizantes das mães), Inter.(P) (internalizantes dos pais), Exter.(P) (externalizantes dos pais).

TABELA 2

Correlações entre as Percepções do Clima Familiar dos Adolescentes e de seus Pais

\begin{tabular}{cccc}
\hline \multicolumn{3}{c}{ Perspectivas } \\
\hline Mapoio & $0,48^{* * *}$ & 0,12 & $0,32 * *$ \\
Hierarquia & $0,35^{* * *}$ & $0,32 * *$ & $0,28^{* * *}$ \\
Coesão & $0,47 * * *$ & $0,32^{* *}$ & $0,35^{* * *}$ \\
Conflito & $0,47 * * *$ & $0,25 *$ & $0,35^{* * *}$ \\
\hline
\end{tabular}

$* \mathrm{p}<0,05, * * \mathrm{p}<0,01, * * * \mathrm{p}<0,0001$.

\section{Correlações entre Problemas Emocionais e de Comportamento e Clima Familiar}

As associações entre os PEC e as relações familiares dos filhos, pais e mães foram investigadas por meio das correlações de Pearson. Os resultados estão na Tabela 3.

Especificamente com relação às comparações intrapessoais (i.e. escores de correlação dos PEC e do clima familiar do mesmo individuo), pode ser observado na Tabela 3 que, com exceção da correlação 
TABELA 3

Problemas Emocionais e de Comportamento dos Pais com a Percepção do Clima Familiar pelos Adolescentes e seus Pais

\begin{tabular}{|c|c|c|c|c|c|c|}
\hline & Inter.(A) & Exter.(A) & Inter.(M) & Exter.(M) & Inter. $(P)$ & Exter. $(P)$ \\
\hline Apoio(A) & $-0,11$ & $-0,19 *$ & $-0,10$ & $-0,09$ & 0,11 & 0,12 \\
\hline Hierarquia(A) & $0,17^{*}$ & $0,32 * * *$ & $0,17^{*}$ & $0,21^{*}$ & 0,08 & 0,09 \\
\hline Coesão(A) & $-0,24 * * *$ & $-0,27 * * *$ & $-0,15$ & $-0,21 *$ & $-0,09$ & 0,05 \\
\hline Conflito(A) & $0,31 * * *$ & $0,42 * * *$ & $0,22 * *$ & $0,32 * *$ & 0,07 & 0,06 \\
\hline Apoio(M) & $-0,17$ & $-0,21 *$ & $-0,24 * *$ & $-0,19^{*}$ & $-0,02$ & 0,07 \\
\hline Hierarquia(M) & $0,17 *$ & 0,12 & $0,17^{*}$ & $0,33 * * *$ & $-0,17$ & 0,15 \\
\hline Coesão(M) & $-0,17^{*}$ & $-0,18^{*}$ & $-0,29 * * *$ & $-0,28 * * *$ & $-0,23^{*}$ & 0,20 \\
\hline Conflito(M) & $0,21 *$ & $0,29 * * *$ & $0,41 * * *$ & $0,55 * * *$ & $0,26^{*}$ & $-0,32 * *$ \\
\hline Apoio(P) & $-0,22 *$ & 0,16 & $-0,30 * *$ & $-0,27^{*}$ & $-0,50 * * *$ & $-0,46^{* * *}$ \\
\hline Hierarquia(P) & $-0,03$ & 0,03 & 0,19 &,$- 24 *$ & $0,35^{* * *}$ & $0,34 * * *$ \\
\hline Coesão(P) & $-0,20$ & $-0,18$ & $-0,32 * * *$ & $-0,37 * * *$ & $-0,64 * * *$ & $-0,63 * * *$ \\
\hline Conflito(P) & 0,18 & $0,22 *$ & 0,18 & $0,27 *$ & $0,64 * * *$ & $0,63 * * *$ \\
\hline
\end{tabular}

$* * \mathrm{p}<0,01, * * * \mathrm{p}<0,001$.

Abreviações: Inter.(A) (internalizante do adolescente), Exter.(A) (externalizante do adolescente), Inter.(M) (internalizante da mãe), Exter.(M) (externalizante da mãe), Inter.(P) (internalizante do pai), Exter.(P) (externalizante do pai). As partes sombreadas referem-se às correlações entre as percepções do sistema familiar e dos problemas emocionais e de comportamento do mesmo participante.

entre sintomas internalizantes e apoio percebidos pelos adolescentes $(\mathrm{r}=-0,11, \mathrm{~ns})$, todas as outras associações foram significativas. De modo geral, os resultados apontam que uma maior intensidade de sintomas internalizantes e externalizantes está positivamente relacionada com baixo apoio e baixa coesão, além de altos níveis de conflito e diferenciação hierárquica entre gerações.

A percepção de altos níveis de sintomas internalizantes e externalizantes pelos adolescentes está fracamente relacionada com algumas percepções familiares apresentadas pelos pais e mães, sendo todas abaixo de 0,30. Com as mães, foram significativas seis das oito correlações possíveis. No caso dos pais, foram significativas duas das oito associações calculadas.

A percepção dos PEC pelas mães correlacionase com cinco das oito dimensões do relacionamento familiar descritas pelos filhos (magnitude variando de $0,17 \mathrm{a} 0,32$ ). No que diz respeito às relações familiares descritas pelos pais, foram seis em oito possíveis (magnitude variando de 0,27 a 0,37). Finalmente, os sintomas descritos pelos pais não se correlacionaram com a descrição familiar feita pelos adolescentes e, com relação à mãe, foram encontradas três associações em oito significativas.

\section{DISCUSSÃO E CONSIDERAÇÕES FINAIS}

O presente estudo buscou investigar os PEC e sua relação com o clima familiar na visão dos adolescentes e de seus pais. Inicialmente, foram analisados os padrões de correlações entre as percepções dos problemas internalizantes e externalizantes dos adolescentes, seus pais e suas mães. Os resultados mostraram correlações moderadas entre a percepção dos problemas internalizantes e externalizantes, que variaram de 0,59 a 0,79 , sugerindo que a percepção das duas sintomatologias (internalizantes e externalizantes) estão relacionadas, podendo aparecer simultaneamente. De acordo com a literatura específica, quando os PEC internalizantes e externalizantes co-ocorrem há uma predominância de fatores ambientais em relação aos biológicos e genéticos (Mun, Fitzgerald, Von Eye, Puttler, \& Zucker, 2001). Dentre os aspectos ambientais que podem contribuir para o aparecimento de PEC em adolescentes estudos tem sugerido a influência das relações familiares e, também, dos conflitos conjugais (Silvares \& Souza, 2008; Teodoro, Cardoso, \& Pereira, 2011).

As comparações entre as percepções dos participantes da pesquisa quanto aos sintomas internalizantes e externalizantes indicaram um claro padrão no qual há uma relação entre a descrição do grupo materno com o paterno (associações variando de 0,31 a 0,41 ) e com os do filho $(0,26$ a 0,37$)$. Não foram encontradas, no entanto, associações entre as percepções dos filhos e dos pais com relação aos PEC. Embora os pais possam estar mais presentes no ambiente familiar, este dado reflete a diferença de padrões de relacionamentos que os filhos estabelecem com as mães e com os pais. Achenbach (1992) postula que pais e mães podem ter percepções diferenciadas sobre os comportamentos dos 
filhos devido às diferenças nas interações parentais, havendo, ainda, uma maior aproximação dos filhos com a figura materna. De acordo com Middleton, Scott e Renk (2009), os adolescentes estabelecem um padrão de relacionamento distinto para com as mães e os pais, fazendo-se necessário, inclusive, a realização de intervenções distintas com pais e mães.

Os problemas internalizantes maternos correlacionaram-se, positivamente, com a percepção de Hierarquia do adolescente e negativamente com a Afetividade. Quanto aos problemas externalizantes, as correlações foram positivas em relação ao Conflito e Hierarquia, e negativas com a Coesão e a Afetividade. Esses dados corroboram os achados de EspositoSmytherset al. (2006), no qual a presença de PEC maternos esteve associada a menor coesão familiar, bem como maior conflito familiar. Convém salientar que, conforme postulado por Feldman e Gehring (1988), por serem os responsáveis pela educação de seus filhos e, consequentemente, pelo estabelecimento de regras no ambiente familiar, é esperado que os pais possuam uma maior hierarquia com relação aos filhos, sendo desejável tal diferença.

Por fim, face à constatação da associação entre os PEC dos pais e a estruturação familiar como um todo, e os PEC dos filhos, demonstrados tanto pelos dados apresentados nesta pesquisa, como pela literatura vigente, faz-se necessário destacar a importância da realização de estudos futuros que investiguem esta temática. O presente estudo não esgota a temática discutida, sendo sugeridos, inclusive, estudos longitudinais, que possam entender melhor esta temática.

\section{REFERÊNCIAS}

Achenbach T. M. (1992). Manual for the Child Behavior Checklist/2-3 and 1992 profile. Burlington, VT: University of Vermont.

Achenbach, T. M. (1991). Manual for the Child Behavior Checklist/ 4-18 e 1991 profile. Burlington, VT: University of Vermont.

Achenbach, T. M., \& Rescorla, L. A. (2001). Manual for the ASEBA school-age forms \& profiles. Burlington: University of Vermont, Research Center for Children, Youth, \& Families.

Achenbach, T. M., \& Rescorla, L. A. (2003). Manual for the ASEBA Adult Forms \& Profiles. Burlington, VT: University of Vermont, Research Center for Children, Youth, \& Families.

Achenbach, T., \& Howell, C. (1993). Are American children's problems getting worse? A 13-year comparison. Journal of American Academy on Child and Adolescent Psychiatry, 32, $1145-1154$.

Adams, R. E., \& Laursen, B. (2007). The correlates of conflict: Disagreement is not necessarily detrimental. Journal of Family Psychology, 21(3), 445-458. doi: 10.1037/0893-3200.21.3.445

Althoff, R. R. (2008). Diagnoses, neuropsychological functioning, and parental depression affect the expression of internalizing and externalizing disorders in children. Journal of the American Academy of Child \& Adolescent Psychiatry, 4, 358-358.
Associação Americana de Psiquiatria (2002). Manual Diagnóstico e Estatístico de Transtornos Mentais - IV. Porto Alegre: ArtMed.

Avanci, J. Q., Assis, S. G., \& Oliveira, R. V. C. (2008). Sintomas depressivos na adolescência: estudo sobre fatores psicossociais em amostra de escolares de um município do Rio de Janeiro, Brasil. Cadernos de Saúde Pública, 24(10), 2334-2346. doi: 10.1590/S0102-311X2008001000014

Benetti, S. P. C. (2006). Conflito Conjugal: Impacto no Desenvolvimento Psicológico da Criança e do Adolescente. Psicologia: Reflexão e Crítica, 19(2), 261-268.

Buehler, C. (2006). Parents and peers in relation to early adolescent problem behavior. Journal of Marriage and Family, 68, 109-124.

Buehler, C., Benson, M., \& Gerard, J. M. (2006). Interparental hostility and early adolescent problem behavior: The mediating role of specific aspects of parenting. Journal of Research on Adolescence, 16, 265-292.

Canino, G., Shrout, P. E, Rubio-Stipec, M., Bird, H. R., Bravo, M., Ramírez, R., Chaves, L., Alegría, M., Bauermeister, J. J., Hohmann, A., Ribera, J., García, P., \& Martínez-Taboas, A. (2004). The DSM-IV rates of child and adolescent disorders in Puerto Rico: Prevalence, correlates, service use, and the effects of impairment. Archives of General Psychiatry, 61, 85-93.

Costello, D. M., Rose, S., Swendsen, J., \& Dierker, L. C. (2008). Risk and protective factors associated with trajectories of depressed mood from adolescence to early adulthood. Journal of Consulting \& Clinical Psychology, 76, 173-183. doi: 10.1037/0022-006X.76.2.173

Cruvinel, M., \& Boruchovitch, E. (2009). Sintomas de depressão infantil e ambiente familiar. Psicologia em Pesquisa-UFJF, 3 , $87-100$.

Davis, K. A., \& Epkins, C. C. (2009). Do private religious practices moderate the relation between family conflict and preadolescents' depression and anxiety symptoms? Journal of Early Adolescence, 29(5), 693-717.

Dorsey, S., Forehand, R., \& Brody, G. (2007). Coparenting conflict and parenting behavior in economically disadvantaged single parent African American families: The role of maternal psychological functioning. Journal of Family Violence, 22, 621-630.

Esposito-Smythers, C., Birmaher, B., Valeri, S., Chiappetta, L., Hunt, J., Ryan, N., Axelson, D., Strober, M., Leonard, H., Sindelar, H. et al. (2006). Child comorbidity, maternal mood disorder, and perceptions of family functioning among bipolar youth. Journal of the American Academy of Child and Adolescent Psychiatry, 45(8), 955-964.

Feldman, S.S., \& Gehring, T.M. (1988). Changing perceptions of family cohesion and power across adolescence. Child Development, 59, 1034-1045.

Féres-Carneiro, T., Henriques, C. R., \&Jablonski, B. (2011). Um jogo interativo: a relação entre pais e filhos adultos no cotidiano familiar contemporâneo. Psico, PUCRS, 42(2), 236-245.

Fleitlich-Bilyk, B., \& Goodman, R. (2001). Social factors associated with child mental health problems in Brazil: Cross sectional survey. British Medical Journal, 323, 599-600. doi: 10.1136/bmj.323.7313.599

Fleitlich-Bilyk, B., \& Goodman, R. (2004), Prevalence of child and adolescent psychiatric disorders in southeast Brazil. Journal of American Academy Child and Adolescence Psychiatry, 43(6), 727-734.

Hackett, R., \& Hackett, L. (1999). Child psychiatry across cultures. International Review of Psychiatry, 11, 225-235. doi: 10.1080/09540269974410

Harkness, K. L., Lumley, M. N., \& Truss, A. E. (2008). Stress generation in adolescent depression: the moderating role of child abuse and neglect. Journal of Abnormal Child Psychology, $36(3), 421-432$. 
Jablonski, B. (2010). A divisão de tarefas domésticas entre homens e mulheres no cotidiano do casamento. Psicologia: Ciência e Profissão, 30(2), 262-275. doi: 10.1590/S141498932010000200004

Jacobs, R. H., Reinecke, M. A., Gollan, J, K., \& Kane, P. (2008). Empirical evidence of cognitive vulnerability for depression among children and adolescents: A cognitive science and developmental perspective. Clinical Psychology Review, 28, 759-782.

Kapi, A., Veltsista, A., Kavadias, G., Lekea, V., \& Bakoula, C. (2007). Social determinants of self-reported emotional and behavioral problems in Greek adolescents. Social Psychiatry and Psychiatric Epidemiology, 42, 594-598. doi: 10.1007/ s00127-007-0201-4

Levandowski, D. C., Koller, S. H., \& Piccinini, C. A. (2002). Paternidade na adolescência e os fatores de risco e de proteção para a violência na interação pai-criança. Interações, 7(13), 77-100.

Marin, A. H., Piccinini, C. A., \& Tudge, J. R. H. (2011). Estabilidade e mudança nas práticas educativas maternas e paternas ao longo dos anos pré-escolares da criança. Psicologia: Reflexão e Crítica, 24(1), 71-79. doi: 10.1590/S0102-79722011000100009

Matud, M. P. (2007). Domestic abuse and children's health in the Canary Islands, Spain. European Psychologist, 12(1), 45-53. doi: 10.1027/1016-9040.12.1.45

Middleton, M., Scott, S. L., \& Renk, K. (2009). Parental depression, parenting behaviours, and behaviour problems in young children. Infant and Child Development, 18(4), 323-336. doi: $10.1002 /$ icd.598

Morawska, A., \& Thompson, E. (2009). The Parent Problem Checklist: Examining the effects of parenting conflict on children. Australian and New Zealand Journal of Psychiatry, 43(3), 260-269.

Mun, E. Y., Fitzgerald, H. E., Von Eye, A., Puttler, L. I., \& Zucker, R. A. (2001). Temperamental characteristics as predictors of externalizing and internalingproblens in the context of high and low parental psychopathology. Infant Mental Health Journal, 22(3), 393-415.

Oliva, A., \& Parra, A. (2004). Contexto Familiar e desarrollo psicológico durante la adolescência. In E. Arranz (Ed.). Familia y desarrollo psicológico (pp. 96-123). Madrid: Pearson Educación.

Osório, L. C. (2002). Terapia de família: novas tendências. Porto Alegre: Artmed.

Parsons, C. (2003). Caring for adolescents and families in crisis. Nursing Clinics of North America, 38(1), 111-122. doi: 10.1016/ S0029-6465(02)00071-3

Pratta, E. M. M., \& Santos, M. A. (2007). Família e adolescência: A influência do contexto familiar no desenvolvimento psicológico de seus membros. Psicologia em Estudo, Maringá, 12(2), 247-256.

Rocha, M. M., Araújo, L. G. S., \& Silvares, E. F. M. (2008). Um estudo comparativo entre duas traduções brasileiras do Inventário de Auto-Avaliação para Jovens (YSR). Psicologia: Teoria e Prática, 10, 14-24.

Sawyer, M. G., Miller-Lewis, L, R., \& Clark, J. J. (2006). The mental health of 13-17 year-olds in Australia: Findings from the national survey of mental health and well-being. Journal of Youth and Adolescence, 36(2), 185-194.
Sheeber, L., Hops, H., Alpert, A., Davis, B., \& Andrews, J. (1997). Family support and conflict: Prospective relations to adolescent depression. Journal of Child Psychology, 25, 333-344. doi: 10.1023/A:1025768504415

Silvares, E. F. M., \& Souza, C. L. (2008). Discórdia conjugal: distúrbios psicológicos infantis e avaliação diagnóstica comportamental-cognitiva. Psicologia: Teoria e Prática, 10, 200-213.

Sourander, A., Niemela, S., Santalahti, I., Helenius, H., \& Piha, J. (2008). Changes in psychiatric problems and service use among 8-year-old children: A 16-year population-based time-trend study. Journal of the American Academy of Child and Adolescent Psychiatry, 47(3), 317-327.

Stark, K. D., Humphrey, L. L., Crook, K., \& Lewis, K. (1990). Perceived family environments of depressed and anxious children: Child's and maternal figure's perspectives. Journal of Abnormal Child Psychology, 18, 527-547.

Stocker, C. M., Richmond, M. K., \& Rhoades, G. K. (2007). Family emotional processes and adolescents' adjustment. Social Development, 16(2), 310-325.

Teodoro, M. L. M., Cardoso, B. M., \& Pereira, T. F. P. (2011). As relações familiares e os problemas emocionais e de comportamento em adolescentes. In Wagner, A e cols. (2011). Desafios Psicossocias da Família Contemporânea: pesquisa e reflexões (pp. 140-149). Porto Alegre: Artmed.

Teodoro, M. L. M., Cardoso, B. M., \& Freitas, A. C. H. (2010). Afetividade e conflito familiar e sua relação com a depressão em crianças e adolescentes. Psicologia: Reflexão e Crítica, 23(2), 324-333. doi: 10.1590/S0102-79722010000200015

Teodoro, M. L. M., Land, B. R., \& Allgayer, M. (2009). Desenvolvimento e validade fatorial do Inventário do Clima Familiar (ICF) para adolescentes. Psicologia: Teoria e Prática, 11(3), 27-39.

Wagner, A., \& Predebon, J. C. (2005). Problemas de comportamento na adolescência: configuração familiar e aspectos sóciodemográficos. Práxis, 2(2), s/n.

\section{Autores:}

Maycoln Leôni Martins Teodoro - Doutor em Psicologia Clínica e do Desenvolvimento (Albert Ludwigs Universität Freiburg, Alemanha). Professor Adjunto I, Universidade Federal de Minas Gerais (UFMG).

Adriana Raquel Binsfeld Hess - Mestre em Psicologia pela Universidade do Vale do Rio dos Sinos. Doutoranda em Psicologia, Universidade Federal do Rio Grande do Sul (UFRGS). Professora Titular, Faculdade de Desenvolvimento do Rio Grande do Sul (FADERGS)

Lisiane Alvim Saraiva - Mestre em Psicologia pela Pontifícia Universidade Católica do Rio Grande do Sul (PUCRS). Doutoranda em Psicologia, Universidade Federal do Rio Grande do Sul (UFRGS).

Bruna Moraes Cardoso - Mestre em Psicologia pela Universidade do Vale do Rio dos Sinos (UNISINOS). Professora na Universidade Regional Integrada do Alto Uruguai e das Missões/Erechim (URI-Erechim).

Endereço para correspondência:

Maycoln Leôni Martins Teodoro

Universidade Federal de Minas Gerais

Faculdade de Filosofia e Ciências Humanas

Departamento de Psicologia

Av. Antonio Carlos, 6627

CEP 31270-930 Belo Horizonte, MG, Brasil

E-mail: mlmteodoro@hotmail.com

Recebido em: 25.04 .2013

Aceito em: 30.05.2014 